\title{
Ischemic Stroke Secondary to Left Ventricular Noncompaction
}

\author{
Hussam A. Yacoub ${ }^{\mathrm{a}, \mathrm{b}, \mathrm{c}}$, Keithan Sivakumar ${ }^{\mathrm{a}}$, Mohammed El-Hunjul ${ }^{\mathrm{a}}$, \\ Chun Chu ${ }^{\mathrm{a}}$, Dev Mehta ${ }^{\mathrm{a}}$
}

\begin{abstract}
Left ventricular noncompaction (LVNC) is a rare cause of cardiomyopathy that can lead to systemic embolism and ischemic stroke. LVNC results from the arrest of ventricular myocardium compaction during embryogenesis. Multiple other cardiac complications coexist with this cardiomyopathy, and one of the potential consequences is cardioembolic events causing ischemic stroke. This condition can be underdiagnosed or misdiagnosed as hypertrophic or dilated cardiomyopathy. We report a patient who presented with recurrent embolic ischemic stroke secondary to LVNC that was overlooked on initial transthoracic echocardiographic studies. After an unremarkable laboratory workup, transesophageal echocardiogram (TEE) revealed noncompaction of the myocardium within the apex of the left ventricle, confirmed by cardiac magnetic resonance imaging (MRI). The patient was anticoagulated with warfarin and discharged to a rehabilitation facility. Increased understanding and awareness of the diagnosis, clinical manifestations, and management of LVNC are advised, particularly in patients with recurrent embolic stroke of undetermined source. Screening of all first-degree relatives with this familial condition is recommended as well, as appropriate treatment can prevent cardiac complications and sudden death.
\end{abstract}

Keywords: Left ventricular noncompaction; Cardiomyopathy; Ischemic stroke; Echocardiography; Cryptogenic stroke

\section{Introduction}

Left ventricular noncompaction (LVNC), first described by Chin et al in 1990 [1], is a cardiomyopathy that is characterized by deep trabeculations of the ventricular wall, with echocardiographic evidence of multiple deep intertrabecular recesses communicating with the ventricular cavity. This occurs as a result of the arrest of ventricular myocardium compaction during

Manuscript submitted September 6, 2019, accepted September 23, 2019

aLehigh Valley Physician Group-Neurology, Lehigh Valley Health Network, Allentown, PA, USA

bMorsani College of Medicine, University of South Florida, Tampa, FL, USA ${ }^{c}$ Corresponding Author: Hussam A. Yacoub, Department of Neurology, Lehigh Valley Health Network, Suite 405, 1250 S. Cedar Crest Blvd., Allentown, PA 18103, USA. Email: hyacoub123@yahoo.com

doi: https://doi.org/10.14740/jnr553 embryogenesis. The exact cause of this rare cardiomyopathy is not well understood. Noncompacted myocardium has been categorized as an unclassified cardiomyopathy by the World Health Organization [2, 3]. LVNC is also known as spongy myocardium, spongiform cardiomyopathy, or hypertrabeculation.

Common coexisting cardiac findings include left-ventricular systolic dysfunction, valvular disease, congestive heart failure, and malignant arrhythmias. Isolated LVNC, a primary genetic cardiomyopathy that is associated with no other congenital cardiac malformations, is extremely rare [1]. Neurological complications include cardioembolic cerebral infarcts, and the association of LVNC with ischemic stroke continues to be underestimated.

The differential diagnosis of LVNC includes apical hypertrophic cardiomyopathy, arrhythmogenic right ventricular dysplasia, and cardiac metastases [4].

Although the exact prevalence of ventricular noncompaction in the general population is not known, it is estimated to range from $0.014 \%$ to $1.3 \%$ [5-7], and is reported in $3-4 \%$ among patients with heart failure [8]. The prevalence among adults who are referred for echocardiography studies is estimated between $0.01 \%$ and $0.27 \%[9,10]$. The incidence is estimated between $0.05 \%$ and $0.25 \%$ per year [11]. The diagnosis can be challenging [12] as prominent trabeculations can be seen in $68 \%$ of healthy individuals [13], and spongy myocardium has to be distinguished from normal muscle bundles.

The most commonly utilized diagnostic tool to evaluate noncompaction cardiomyopathy is echocardiography. There are several echocardiogram criteria that must be met to make the diagnosis of LVNC, including the presence of spongy noncompacted myocardium and visualization of intertrabecular spaces perfusion from the ventricular cavity [11]. If LVNC is suspected on transthoracic echocardiogram (TTE) or transesophageal echocardiogram (TEE), cardiac magnetic resonance imaging (MRI) can be done for confirmation, which carries a very high specificity [14].

In this report, we present a rare case of recurrent embolic ischemic stroke secondary to LVNC that was overlooked on initial surface echocardiogram but was better revealed on transesophageal echocardiography and cardiac MRI. The patient was eventually started on warfarin and discharged in a stable condition to a rehabilitation facility. We also elaborate on the epidemiology, clinical manifestation, diagnostic criteria, and management of this rare cardiomyopathy.

\section{Case Report}

A right-handed 39-year-old African American man was 
brought to the emergency department after being found on the floor by a friend. On initial evaluation, the patient was found to have left-sided hemiplegia and right gaze preference, along with hemineglect.

One year prior, the patient presented to a local hospital with left-sided visual loss and was found to have a right occipital infarct with residual left-sided visual field cut. He was discharged on daily aspirin. Two months later, he presented to our hospital with chest pain; workup revealed severe congestive heart failure with an ejection fraction of $15-20 \%$ and a left ventricular thrombus. At that time, he was started on warfarin but was noncompliant.

Past medical history was significant for hypertension and type 2 diabetes mellitus; however, the patient was not compliant with his medications. He had a history of alcohol, cocaine, and heroin abuse but denied the use of any recently. Family history revealed that both his parents died in their early $50 \mathrm{~s}$ secondary to coronary artery disease.

On physical examination, the patient was afebrile with a blood pressure of 216/164 and a heart rate of 83 beats per minute. He was in normal sinus rhythm. On neurological examination, he was found to be awake and alert with no aphasia or dysarthria. Cranial nerve examination revealed a right gaze preference, left homonymous hemianopia, and left central facial weakness. Motor strength was $5 / 5$ on the right side and $0 / 5$ in the left arm and leg, best described as flaccid paralysis. Sensory examination revealed diminished sensation to pinprick on the left. Further examination revealed marked sensory extinction on the left and profound left-sided visuospatial hemineglect.

Laboratory workup included a complete blood count, metabolic panel, and a urine drug screen, all of which were unremarkable. International normalized ratio was 1.0. A computed tomography of the head showed acute ischemia in the right posterior frontal, temporal, and parietal lobes involving gray and white matter. The patient was deemed not a candidate for intravenous thrombolytics or mechanical thrombectomy as the stroke was well formed and extensive on the initial imaging studies.

MRI of the brain showed a large acute ischemic infarct in the territory of the right middle cerebral artery (MCA). Electrocardiogram and telemetry did not show any atrial fibrillation. Magnetic resonance angiography showed occlusion of the M1 segment of the right MCA. Since a TTE was done during a recent hospitalization, a TEE was completed and revealed severe left ventricular dilation and hypokinesis with no evidence of thrombus, an ejection fraction of $15 \%$, and right ventricular wall hypokinesis. Intravenous contrast was injected to better visualize the left ventricle showing hypertrabeculation of the apex. Cardiac MRI revealed noncompaction of the myocardium within the apex of the left ventricle (Fig. 1), but no thrombi were seen. The patient was evaluated by cardiology and was found to meet all the diagnostic criteria of LVNC.

To reduce the risk of hemorrhagic conversion of a largeterritory infarct, anticoagulation was not immediately initiated. Patient was started on pharmacotherapy for the systolic dysfunction. Cardiology recommended an implantable cardioverter-defibrillator, which was inserted before his discharge. The patient was deemed clinically stable and eventually discharged

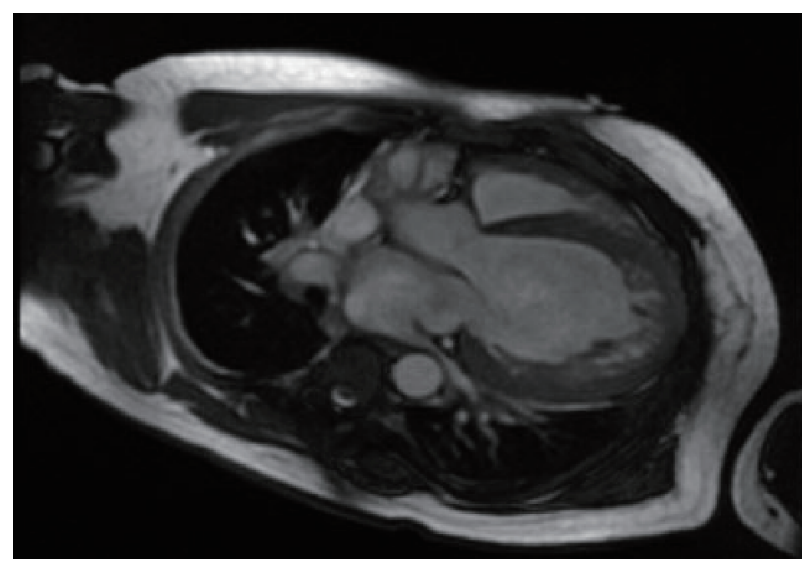

Figure 1. Cardiac MRI revealing left ventricular noncompaction. MRI: magnetic resonance imaging.

to a rehabilitation facility on warfarin.

\section{Discussion}

LVNC is a cardiomyopathy characterized by remarkable trabeculae in the ventricles with deep intertrabecular recesses between that result in cardiac dysfunction and multiple complications. These trabecular arrangements are readily distinguishable from the trabeculations seen in other types of cardiomyopathy [15]. LVNC was first described in 1932 in an infant heart that was histologically studied. Findings were described as the remains of the sinusoids of the embryonic hearts [16]. The most widely accepted theory of the pathogenesis of LVNC is that compaction gets arrested or impaired leaving the remains of the recesses $[1,17,18]$. Others have suggested that alternative pathologies such as myocardial dissection might be implicated [9].

The term hypertrophic LVNC indicates the coexistence of LVNC with hypertrophic cardiomyopathy; two cases were recently reported after diagnosis based on echocardiography and cardiac magnetic imaging [19]. Another rare entity is the combination of left ventricular hypertrophy and noncompaction [20].

\section{Epidemiology}

The prevalence of LVNC in patients undergoing echocardiography is rare. In a study conducted by the Mayo Clinic [5], the prevalence was found to be $0.02 \%$ after 141,047 echocardiograms were obtained in patients between 2001 and 2006. It is estimated that $0.014-1.3 \%$ of the general population have LVNC [5-7], and the prevalence is estimated to be $0.01-0.27 \%$ in those who have had echocardiography $[10,11,21,22]$. In several cohorts of subjects with ventricular noncompaction, the percentage of men was reported as ranging from $56 \%$ to $82 \%[1,5,11,23,24]$. In a study of more than 900 patients with heart failure, the prevalence of LVNC was found to be $3 \%[8]$. 


\section{Clinical manifestations and the incidence of ischemic stroke}

It is well known that LVNC is associated with cardioembolic events. The rate of ischemic stroke occurrence appears to be dependent on age, cardiovascular comorbidities, and duration of follow-up [23]. In one study by Stollberger et al [25], the occurrence of systemic embolization, including stroke, was $10 \%$ in patients with LVNC compared to $15 \%$ in controls, and therefore LVNC was not considered a risk factor for cardioembolism. Due to the low prevalence of LVNC in the general population, the incidence of cryptogenic stroke is not known [26].

A systemic overview study of 241 patients with LVNC aimed to examine the natural history and clinical manifestations of this condition [27]. It was found that dyspnea was the most common baseline characteristic, with $3 \%$ having had a prior stroke. After 39 months of follow-up, $8 \%$ had a stroke or transient ischemic attack; most patients were not on anticoagulation. Atrial fibrillation was present in $10 \%$ of patients. The overall mortality at 39 months was $14 \% ; 50 \%$ of those died as a result of sudden cardiac arrest [27].

\section{Diagnostic criteria}

Several authors have reported the likelihood of missing the diagnosis of LVNC and emphasized the importance of awareness of this condition $[9,28]$. The diagnosis can be overlooked depending on image quality and interpretation. Failure to diagnose may lead to insufficient treatment and management as this condition can lead to malignant arrhythmias and sudden death. Surface echocardiogram remains the most commonly ordered initial test that identifies the characteristic findings of LVNC. A TEE can be obtained if there is a suspicion and diagnosis was not made on TTE. In our patient, LVNC was overlooked on the initial transthoracic echocardiographic study but was better distinguished on TEE.

Several echocardiographic criteria were proposed to aid in the diagnosis of LVNC in the literature. These include criteria described by Chin [1], Jenni [17, 18], and Stollberger [29]. After review of all criteria, we find the revised criteria by Stollberger et al [30] reported after a review of 115 echocardiograms by three experts of LVNC the most ideal. The criteria are: 1) Greater than three prominent trabecular formations along the left ventricular endocardial border, which can be seen at end-diastole; 2) Trabeculations move synchronously with the compacted myocardium; 3) Trabeculations make up the noncompacted part of the two-layer myocardial structure, best seen at end-systole; 4) Perfusion of the intertrabecular spaces from the ventricular cavity is present at end-diastole on color or contrast echocardiography [30].

Cardiac MRI can also be of diagnostic value when adequate echocardiographic images cannot be obtained and in cases that are highly suspicious for LVNC but not confirmed. Cardiac MRI has a higher sensitivity, especially in detecting trabeculation and recesses at the apex and lateral wall [14], and allows the differentiation of compacted and noncompacted myocardium. Peterson et al suggests that a ration of noncom- pacted/compacted myocardium of $>2.3$ in diastole is highly suggestive of LVNC [14]. Boban et al [31] further evaluated the benefit of cardiac MRI in the diagnosis of LVNC and verified its high specificity and sensitivity. Another advantage of cardiac MRI is the benefit of imaging the apex and the use of enhancement to evaluate fibrosis $[32,33]$ and superior visualization of left ventricular thrombi [33]. Left ventricular angiography is rarely essential to make the diagnosis [33].

\section{Stroke prevention}

The use of anticoagulation for stroke prevention in patients with LVNC appears to be controversial. Some authors recommend using chronic anticoagulation for all patients with LVNC as a primary prevention of stroke [8]. In a retrospective study by Stollberger et al [34], a high $\mathrm{CHA}_{2} \mathrm{DS}_{2}$-VASc score was associated with increased incidence of cardioembolism, an observation that may help in guiding patient management and stroke prevention. In recent studies, anticoagulation has been recommended in patients with LVNC with severe systolic dysfunction (ejection fraction of $<40 \%$ ), atrial fibrillation, recurrent embolic events, or presence of ventricular thrombi $[6,35$, 36].

A retrospective study of 169 patients evaluated the correlation of high $\mathrm{CHA}_{2} \mathrm{DS}_{2}$-VASc score with cardioembolic events; 24 patients had ischemic stroke, 18 of which were due to cardioembolic events. It was concluded that $\mathrm{CHA}_{2} \mathrm{DS}_{2}$-VASc may aid in making the decision whether to use anticoagulation in patients with LVNC [34].

The use of factor Xa inhibitors for stroke prevention in patients with LVNC has not been studied. Recently, Sun et al [37] reported a case of a 43-year-old man with LVNC and an intraventricular thrombus who was treated with rivaroxaban, $10 \mathrm{mg}$ daily, for 3 months. Follow-up evaluation revealed that the thrombus diminished, but this outcome does not support the use of this class of medication for stroke prevention in patients with LVNC. Further studies are still warranted.

Table $1[5,6,11,23,26,35,38-42]$ summarizes the clinical features and thromboembolic events rate and the use of anticoagulation in adult patients with LVNC reported in several case series.

Our case report demonstrates the potential of underdiagnosis of LVNC in patients with cryptogenic stroke. It also emphasizes the importance of utilization of echocardiographic studies and the need for increased awareness of this entity, particularly in patients with recurrent embolic stroke of undetermined source. If LVNC is suspected on echocardiographic studies, a cardiac MRI is recommended for confirmation. In the case of our patient, the patient had severe systolic dysfunction and establishing a causal relationship between LVNC and ischemic stroke would be complex.

\section{Conclusions}

LVNC is a cardiomyopathy that is characterized by echocardiographic evidence of multiple deep intertrabecular recesses communicating with the ventricular cavity that increases the 


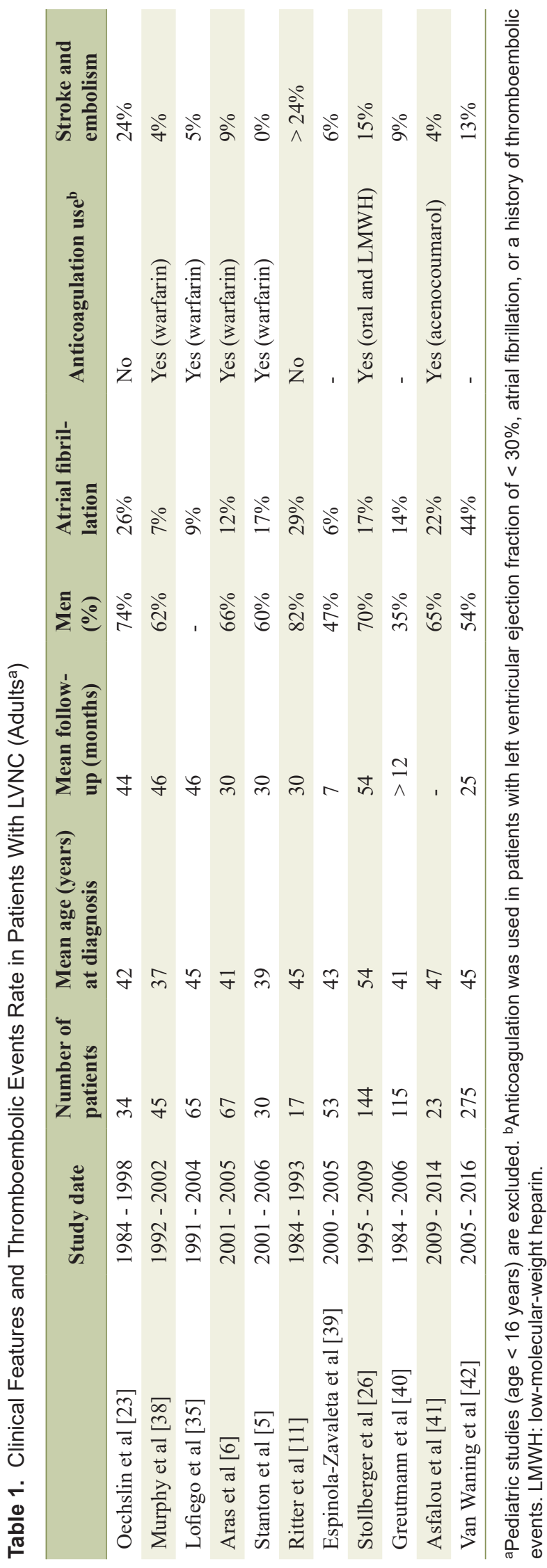

risk of cardioembolic events including ischemic stroke. Surface echocardiographic studies in our patient with recurrent ischemic stroke did not reveal ventricular trabeculations or LVNC, but a follow-up TEE did. Cardiac MRI appears to be highly specific and sensitive in confirming the diagnosis. The use of anticoagulation is controversial for stroke prevention, but seems to be favorable in patients with a high $\mathrm{CHA}_{2} \mathrm{DS}_{2}$ VASc score, atrial fibrillation, and/or recurrent cardioembolic events. We advise clinicians to be aware of this rare cardiomyopathy, particularly in patients with embolic stroke of an unclear source and echocardiogram evidence of ventricular trabeculations.

\section{Acknowledgments}

The authors thank Debra Goldberg for manuscript editing and formatting.

\section{Financial Disclosure}

No external funding was received in support of this work.

\section{Conflict of Interest}

The authors declare that there is no conflict of interest regarding the publication of this paper.

\section{Informed Consent}

Not applicable.

\section{Author Contributions}

All authors had access to the data and a role in writing the manuscript, article type, key words, and running head.

\section{Abbreviations}

LVNC: left ventricular noncompaction; MCA: middle cerebral artery; MRI: magnetic resonance imaging; TEE: transesophageal echocardiogram; TTE: transthoracic echocardiogram

\section{References}

1. Chin TK, Perloff JK, Williams RG, Jue K, Mohrmann R. Isolated noncompaction of left ventricular myocardium. A study of eight cases. Circulation. 1990;82(2):507-513.

2. Richardson P, McKenna W, Bristow M, Maisch B, Mautner B, O'Connell J, Olsen E, et al. Report of the 1995 World Health Organization/International Society and Federation of Cardiology Task Force on the definition 
and classification of cardiomyopathies. Circulation. 1996;93(5):841-842.

3. Elliott P, Andersson B, Arbustini E, Bilinska Z, Cecchi F, Charron P, Dubourg O, et al. Classification of the cardiomyopathies: a position statement from the European Society Of Cardiology Working Group on Myocardial and Pericardial Diseases. Eur Heart J. 2008;29(2):270-276.

4. Zaragoza MV, Arbustini E, Narula J. Noncompaction of the left ventricle: primary cardiomyopathy with an elusive genetic etiology. Curr Opin Pediatr. 2007;19(6):619627.

5. Stanton C, Bruce C, Connolly H, Brady P, Syed I, Hodge $\mathrm{D}$, Asirvatham $\mathrm{S}$, et al. Isolated left ventricular noncompaction syndrome. Am J Cardiol. 2009;104(8):11351138.

6. Aras D, Tufekcioglu O, Ergun K, Ozeke O, Yildiz A, Topaloglu S, Deveci B, et al. Clinical features of isolated ventricular noncompaction in adults long-term clinical course, echocardiographic properties, and predictors of left ventricular failure. J Card Fail. 2006;12(9):726-733.

7. Pignatelli RH, McMahon CJ, Dreyer WJ, Denfield SW, Price J, Belmont JW, Craigen WJ, et al. Clinical characterization of left ventricular noncompaction in children: a relatively common form of cardiomyopathy. Circulation. 2003;108(21):2672-2678.

8. Kovacevic-Preradovic T, Jenni R, Oechslin EN, Noll G, Seifert B, Attenhofer Jost CH. Isolated left ventricular noncompaction as a cause for heart failure and heart transplantation: a single center experience. Cardiology. 2009;112(2):158-164.

9. Stollberger C, Finsterer J. Left ventricular hypertrabeculation/noncompaction. J Am Soc Echocardiogr. 2004;17(1):91-100.

10. Benjamin MM, Khetan RA, Kowal RC, Schussler JM. Diagnosis of left ventricular noncompaction by computed tomography. Proc (Bayl Univ Med Cent). 2012;25(4):354356.

11. Ritter M, Oechslin E, Sutsch G, Attenhofer C, Schneider J, Jenni R. Isolated noncompaction of the myocardium in adults. Mayo Clin Proc. 1997;72(1):26-31.

12. Stollberger C, Finsterer J, Sodeck GH, Grassberger M, Zimpfer D. Stroke from noncompaction overlooked by echocardiography. Int J Cardiol. 2011;148(3):357-358.

13. Boyd MT, Seward JB, Tajik AJ, Edwards WD. Frequency and location of prominent left ventricular trabeculations at autopsy in 474 normal human hearts: implications for evaluation of mural thrombi by two-dimensional echocardiography. J Am Coll Cardiol. 1987;9(2):323-326.

14. Petersen SE, Selvanayagam JB, Wiesmann F, Robson $\mathrm{MD}$, Francis JM, Anderson RH, Watkins H, et al. Left ventricular non-compaction: insights from cardiovascular magnetic resonance imaging. J Am Coll Cardiol. 2005;46(1):101-105.

15. Freedom RM, Yoo SJ, Perrin D, Taylor G, Petersen S, Anderson RH. The morphological spectrum of ventricular noncompaction. Cardiol Young. 2005;15(4):345-364.

16. Bellet S, Gouley BA. Congenital heart disease with multiple cardiac abnormalities: report of a case showing aortic atresia, fibrous scar in myocardium and embryonal sinusoidal remains. Am J Med Sci. 1932;183(4):458-465.

17. Jenni R, Oechslin E, Schneider J, Attenhofer Jost C, Kaufmann PA. Echocardiographic and pathoanatomical characteristics of isolated left ventricular non-compaction: a step towards classification as a distinct cardiomyopathy. Heart. 2001;86(6):666-671.

18. Jenni R, Rojas J, Oechslin E. Isolated noncompaction of the myocardium. N Engl J Med. 1999;340(12):966-967.

19. Tang X, Yu S, Yin L, Gong L. Two Patients with Coincident Noncompacted Myocardium and Hypertrophic Cardiomyopathy. Int Heart J. 2018;59(2):424-426.

20. Misumi I, Honda T, Ishii M, Ohmori K. Coexistence of left ventricular hypertrophy and noncompaction: a case report. J Echocardiogr. 2018;16(2):95-97.

21. Weiford BC, Subbarao VD, Mulhern KM. Noncompaction of the ventricular myocardium. Circulation. 2004;109(24):2965-2971.

22. Ozkutlu S, Ayabakan C, Celiker A, Elshershari H. Noncompaction of ventricular myocardium: a study of twelve patients. J Am Soc Echocardiogr. 2002;15(12):15231528.

23. Oechslin EN, Attenhofer Jost CH, Rojas JR, Kaufmann PA, Jenni R. Long-term follow-up of 34 adults with isolated left ventricular noncompaction: a distinct cardiomyopathy with poor prognosis. J Am Coll Cardiol. 2000;36(2):493-500.

24. Ichida F, Hamamichi Y, Miyawaki T, Ono Y, Kamiya T, Akagi T, Hamada $\mathrm{H}$, et al. Clinical features of isolated noncompaction of the ventricular myocardium: long-term clinical course, hemodynamic properties, and genetic background. J Am Coll Cardiol. 1999;34(1):233-240.

25. Stollberger C, Finsterer J. Left ventricular hypertrabeculation/noncompaction and stroke or embolism. Cardiology. 2005;103(2):68-72.

26. Stollberger C, Blazek G, Dobias C, Hanafin A, Wegner C, Finsterer J. Frequency of stroke and embolism in left ventricular hypertrabeculation/noncompaction. Am J Cardiol. 2011;108(7):1021-1023.

27. Bhatia NL, Tajik AJ, Wilansky S, Steidley DE, Mookadam F. Isolated noncompaction of the left ventricular myocardium in adults: a systematic overview. J Card Fail. 2011;17(9):771-778.

28. Tamborini G, Pepi M, Celeste F, Muratori M, Susini F, Maltagliati A, Veglia F. Incidence and characteristics of left ventricular false tendons and trabeculations in the normal and pathologic heart by second harmonic echocardiography. J Am Soc Echocardiogr. 2004;17(4):367374.

29. Stollberger C, Finsterer J, Blazek G. Left ventricular hypertrabeculation/noncompaction and association with additional cardiac abnormalities and neuromuscular disorders. Am J Cardiol. 2002;90(8):899-902.

30. Stollberger C, Gerecke B, Finsterer J, Engberding R. Refinement of echocardiographic criteria for left ventricular noncompaction. Int J Cardiol. 2013;165(3):463-467.

31. Boban M, Pesa V, Beck N, Manola S, Zulj M, Rotim A, Vcev A. Supplementary diagnostic landmarks of left ventricular non-compaction on magnetic resonance imaging. Yonsei Med J. 2018;59(1):63-71. 
32. Bennett CE, Freudenberger R. The current approach to diagnosis and management of left ventricular noncompaction cardiomyopathy: review of the literature. Cardiol Res Pract. 2016;2016:5172308.

33. Singh DP, Patel H. Left Ventricular Non-compaction (LVNC) Cardiomyopathy. In: StatPearls. Treasure Island (FL), 2019.

34. Stollberger C, Wegner C, Finsterer J. CHADS2- and CHA2DS2VASc scores and embolic risk in left ventricular hypertrabeculation/noncompaction. J Stroke Cerebrovasc Dis. 2013;22(6):709-712.

35. Lofiego C, Biagini E, Pasquale F, Ferlito M, Rocchi G, Perugini E, Bacchi-Reggiani L, et al. Wide spectrum of presentation and variable outcomes of isolated left ventricular non-compaction. Heart. 2007;93(1):65-71.

36. Oechslin E, Jenni R. Left ventricular non-compaction revisited: a distinct phenotype with genetic heterogeneity? Eur Heart J. 2011;32(12):1446-1456.

37. Sun H, Zhao Q, Wang Y, Lakin R, Feng H, Fan X, Luo H, et al. Daily $10 \mathrm{mg}$ rivaroxaban as a therapy for ventricular thrombus related to left ventricular non-compaction cardiomyopathy: A case report. Medicine (Baltimore). 2018;97(4):e9670.
38. Murphy RT, Thaman R, Blanes JG, Ward D, Sevdalis E, Papra E, Kiotsekoglou A, et al. Natural history and familial characteristics of isolated left ventricular non-compaction. Eur Heart J. 2005;26(2):187-192.

39. Espinola-Zavaleta N, Soto ME, Castellanos LM, JativaChavez S, Keirns C. Non-compacted cardiomyopathy: clinical-echocardiographic study. Cardiovasc Ultrasound. 2006;4:35.

40. Greutmann M, Mah ML, Silversides CK, Klaassen S, Attenhofer Jost CH, Jenni R, Oechslin EN. Predictors of adverse outcome in adolescents and adults with isolated left ventricular noncompaction. Am J Cardiol. 2012;109(2):276-281.

41. Asfalou I, Boulaamayl S, Raissouni M, Mouine N, Sabry M, Kheyi J, Doghmi N, et al. Left ventricular noncompaction-A rare form of cardiomyopathy: Revelation modes and predictors of mortality in adults through 23 cases. J Saudi Heart Assoc. 2017;29(2):102-109.

42. van Waning JI, Caliskan K, Hoedemaekers YM, van Spaendonck-Zwarts KY, Baas AF, Boekholdt SM, van Melle JP, et al. Genetics, clinical features, and long-term outcome of noncompaction cardiomyopathy. J Am Coll Cardiol. 2018;71(7):711-722. 